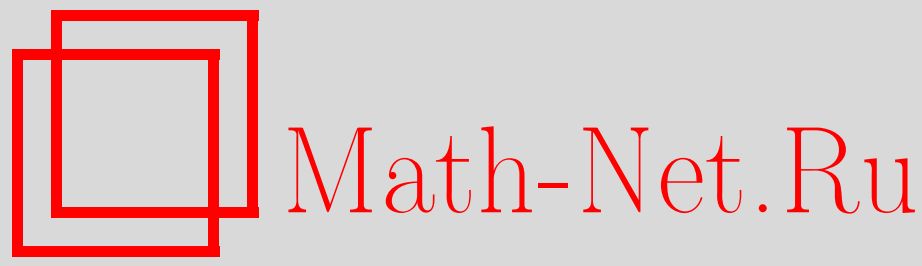

Н. А. Тюрин, Псевдоторические структуры на гиперплоском сечении торического многообразия, ТМФ, 2015, том 182, номер 2, 195-212

DOI: https://doi.org/10.4213/tmf8684

Использование Общероссийского математического портала Math-Net.Ru подразумевает, что вы прочитали и согласны с пользовательским соглашением http://www.mathnet.ru/rus/agreement

Параметры загрузки:

IP : 44.207 .124 .84

26 апреля 2023 г., 16:12:04

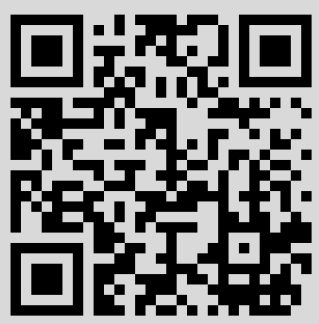




\title{
ПСЕВДОТОРИЧЕСКИЕ СТРУКТУРЫ НА ГИПЕРПЛОСКОМ СЕЧЕНИИ ТОРИЧЕСКОГО МНОГООБРАЗИЯ
}

\begin{abstract}
Продолжены исследования псевдоторической геометрии, а именно представлен тип неторических многообразий, обладающих псевдоторическими структурами. В качестве приложения строится новая псевдоторическая структура на двумерной комплексной квадрике.
\end{abstract}

Ключевые слова: торическое многообразие, псевдоторическая структура, проективное пространство, гиперплоское сечение, интегрируемая система.

DOI: $10.4213 / \operatorname{tmf} 8684$

\section{1. ВВЕДЕНИЕ}

Торическая геометрия есть геометрия фазовых пространств вполне интегрируемых классических механических систем, обладающих свойством регулярности или эффективности (термин “эффективность” в математической литературе в последнее время заменяет оборот “существование переменных действие-угол"). Как оказалось, все типы торических многообразий однозначно определяются комбинаторными данными, а именно выпуклыми многогранниками [1]. То есть геометрия фазовых пространств эффективных вполне интегрируемых систем допускает исчерпывающее и "компактное" описание. Псевдоторическая геометрия есть некоторое обобщение торической геометрии: здесь рассматриваются фазовые пространства с неполными наборами коммутирующих первых интегралов, которые, однако, допускают дополнительную структуру. А именно (см. работу [2]), существуют “вполне интегрируемые листы" неполного набора первых интегралов, т. е. существует семейство симплектических подмногообразий, сохраняющихся гамильтоновым действием неполного набора, и при этом семейство параметризуется некоторым торическим многообразием. Таким образом, псевдоторическая геометрия может пониматься как

*Лаборатория теоретической физики им. Н. Н. Боголюбова, Объединенный институт ядерных исследований, Дубна, Московская обл., Россия. E-mail: ntyurin@theor.jinr.ru

†Факультет математики, Национальный исследовательский университет "Высшая школа экономики", Москва, Россия 
геометрия фазовых пространств интегрируемых систем, близких к вполне интегрируемым системам, но не являющихся таковыми. При этом псевдоторическая структура на фазовом пространстве так же, как и торическая структура во вполне интегрируемом случае, позволяет находить решения классической системы: неполный набор коммутирующих первых интегралов может быть дополнен недостающими особыми интегралами (функциями с особенностями в выделенном базисном множестве, коммутирующими между собой и с исходными интегралами). Из-за особенностей дополнительных интегралов возникают новые типы траекторий, располагающихся на вырожденных торах Лиувилля и других лагранжевых подмногообразиях, которые образованы (как и во вполне интегрируемом случае) совместными множествами уровней регулярных и особых первых интегралов. С точки зрения классической механики псевдоторические структуры оказываются не менее полезными, чем торические.

В качестве примера неэффективной вполне интегрируемой системы приведем систему Гельфанда-Цейтлина на многообразиях флагов [3]. В отличие от эффективной системы, первые интегралы системы Гельфанда-Цейтлина не являются морсовскими функциями, и существует совместное критическое множество уровней, представляющее собой сферу, т. е. стационарные точечные решения составляют большое подмногообразие в фазовом пространстве. В работе [3] была построена псевдоторическая структура на многообразии флагов $F^{3}$, которая предполагает следующую схему описания вполне интегрируемой структуры: первые интегралы делятся на эффективные (каковых на $F^{3}$ два) и индуцированные подъемами с базы псевдоторической структуры. Такие поднятые интегралы являются особыми, однако особенности могут быть разрешены, и после подходящего сглаживания мы получаем гладкие первые интегралы, но эффективность при этом недостижима вследствие топологических препятствий. Таким образом, псевдоторическая геометрия пытается объяснить неэффективную интегрируемость систем и найти топологические условия на фазовые пространства таких систем.

Однако геометрия и топология многообразий, допускающих псевдоторические структуры, пока что очень далека от геометрии торической - даже в найденных примерах неторических псевдоторических многообразий не очень понятно, как комбинаторно описать данные псевдоторических структур. Таким образом, псевдоторическая геометрия пока остается областью эксперимента, в которой в ручном режиме были обнаружены некоторые псевдоторические многообразия, но не было выработано общих законов, которым эти многообразия должны следовать.

В настоящей работе представлен достаточно широкий класс многообразий, обладающих псевдоторическими структурами. Этот класс возникает следующим естественным образом. Всякое гладкое компактное торическое многообразие $X$ может быть вложено в проективное пространство, $\phi: X \rightarrow \mathbb{C P}^{N}$, и общая гиперплоскость $H \subset \mathbb{C P}^{N}$ вырезает в образе вложения подмногообразие комплексной коразмерности 1. Выпуклый многогранник $P_{X}$ при этом оказывается вложенным в выпуклый многогранник $P_{\mathbb{C P}^{N}}$ проективного пространства, вершины которого соответствуют стационарным точкам торического действия $p_{0}, \ldots, p_{N} \subset \mathbb{C P}^{N}$, на которые натянут "базисный симплекс" - прообраз многогранника $P_{\mathbb{C P}^{N}}$ при отображении действия. Для общей гиперплоскости подмногообразие $\phi(X) \cap H$ гладкое; имеется 
естественная стратификация в пространстве гиперплоскостей по числу точек из набора $p_{0}, \ldots, p_{N}$, принадлежащих $H$. Так как гиперплоскости в $\mathbb{C P}^{N}$ описываются точками двойственного пространства $\left(\mathbb{C P}^{N}\right)^{\vee}$, стратификация по этому числу соответствует принадлежности $H$ как точки из пространства $\left(\mathbb{C P}^{N}\right)^{\vee}$ объединению подсимплексов размерности $k$, равной числу точек минус один, двойственного базисного симплекса. При этом существует "порог особости" пересечения $\phi(X) \cap H$, выше которого это пересечение перестает быть гладким. Например, если взять самый маленький страт, состоящий из базисных гиперплоскостей вида $H=\left\{z_{i}=0\right\}$, то пересечение будет особым, но торическим. Следующий страт, соответствующий гиперплоскостям вида $H=\left\{z_{i}+z_{j}=0\right\}$, снова будет соответствовать высечению торического подмногообразия, но на этот раз оно может оказаться гладким. Следующий страт, содержащий плоскости вида $H=\left\{z_{i}+z_{j}+z_{k}=0\right\}$, уже выведет нас из рамок торической геометрии, но может оказаться, что пересечение $\phi(X) \cap H$ обладает псевдоторической структурой.

Этот подход является вполне естественным с точки зрения классической механики. Гиперплоское сечение торического многообразия - это подмногообразие в фазовом пространстве вполне интегрируемой системы, которое может естественно возникнуть в конкретной задаче (например, в рамках теории со связями Дирака), при этом (в случае связи второго рода) получающееся фазовое подпространство уже не будет фазовым пространством вполне интегрируемой системы. Ниже мы увидим, что если сечение не очень общее, то высекаемое фазовое пространство обладает псевдоторической структурой, т. е. соответствующая система, не будучи вполне интегрируемой, очень близка к таковой. Однако главные наши интересы не ограничиваются вопросами классической механики: речь, конечно, идет о квантовании классических механических систем с компактными фазовыми пространствами в терминах лагранжевой геометрии. Для реализации программы лагранжева квантования необходима информации о лагранжевых подмногообразиях фазовых пространств. Как оказалось, псевдоторическая геометрия позволяет строить новые типы лагранжевых подмногообразий и лагранжевых слоений (см. работу [4]), что полезно и для геометрического квантования, и для исследования зеркальной симметрии.

В настоящей статье представлено комбинаторное описание возникновения псевдоторической структуры на многообразии $K_{H}=\phi(X) \cap H$ : вся информация содержится в комбинаторике вложения $P_{X} \subset P_{\mathbb{C P}^{N}}$. Если $X$ само не является проективным пространством, то вложение строгое, но при этом существует минимальное вложение, когда множество вершин $V\left(P_{X}\right)$ совпадает с множеством вершин $V\left(P_{\mathbb{C P}^{N}}\right)$. Поскольку вложение строгое, множества ребер $E\left(P_{X}\right)$ и $E\left(P_{\mathbb{C P}^{N}}\right)$ не совпадают. Обозначим дополнение $E\left(P_{\mathbb{C P}^{N}}\right) \backslash E\left(P_{X}\right)$ как $E_{0}$, при этом заметим, что $E_{0}$ в любом случае не пусто. Найдем максимальный симплекс $\Delta^{k} \subset P_{\mathbb{C P}^{N}}$ размерности $k$, натянутый на ребра из $E_{0}$. Если $k>1$, то конструкция, представленная ниже, показывает, что симплексу $\Delta^{k}$ соответствует иерархия псевдоторических структур на гиперплоском сечении $K_{H}$, т. е. на пересечениях $\phi(X) \cap H$ мы одновременно можем построить псевдоторические структуры рангов $k-1, k-2, \ldots, 0$, причем псевдоторическая структура ранга $i$ будет получаться при рассмотрении гиперплоскости $H$, содержащей ровно $N-i-1$ точек из $\left\{p_{0}, \ldots, p_{N}\right\}$, таким образом, $H$ содержится в страте размерности $i$ (естественно, $i<k$ ). При убывании $i$ в какой-то момент мы пересечем 
порог особости, и наши подмногообразия $K_{H}$ уже не будут гладкими, однако именно этот переход позволяет описать деформации псевдоторических структур на гладких многообразиях в торические структуры на торических вырождениях (пример этого процесса обсуждался в статье [2]).

Построенные псевдоторические структуры можно выстроить по порядку следующим образом: если найдется симплекс $\Delta^{k}, k>1$, описанный выше, то первым делом по нему строится псевдоторическая структура ранга $k-1$ с базой $\mathbb{C P}^{k-1}$ на гиперплоском сечении $K_{H}$, которое соответствует гиперплоскости $H$, проходящей через $N-k$ стационарных точек. Обозначим такой тип гиперплоскоского сечения как $K_{H_{k}}$. Далее, любой подсимплекс $\Delta^{k-1} \subset \Delta^{k}$ обладает теми же определяющими свойствами, что и $\Delta^{k}$, кроме значения размерности. Если $k=2$, то по $\Delta^{k-1}$ строится торическое гиперплоскоское сечение. При этом торическую структуру на $K_{H_{k-1}}$ можно получить деформацией псевдоторической структуры на $K_{H_{k}}$ : построим семейство $K_{H_{k}(t)}, t \in[0,1)$, так что предельной точкой этого семейства будет $K_{H_{k-1}}$. Возможно, при этом мы преодолеваем порог особости, но на данном этапе нас это не интересует. Главное, что псевдоторическая структура на $K_{H_{k}}$ оказывается тесно связанной с торической структурой на $K_{H_{k-1}}$ : например, множество отображений моментов первой структуры естественно дополняется еще одним отображением до полного набора первых интегралов.

Если $k>2$, то $\Delta^{k-1}$ снова дает псевдоторическую структуру, но уже меньшего ранга, база которой содержится в базе начальной псевдоторической структуры, а набор первых интегралов снова является пополнением набора интегралов начальной псевдоторической структуры. Далее мы исчерпываем размерность до тех пор, пока $k-i$ не станет равным 1 и мы получим конечное торическое многообразие $K_{H_{1}}$. В итоге оказывается возможным построить путь в пространстве гиперплоских сечений $K_{H}(t), t \in[0, k-1]$, такой, что соответствующие подмногообразия $K_{H(t)}$ несут на себе псевдоторические структуры ранга $k-[t]-1$, при этом базы этих псевдоторических структур составляют флаг в проективном пространстве $\mathbb{C P}^{k-1}$, а пространство первых интегралов торического многообразия $K_{H(k-1)}=K_{H_{1}}$ при убывании $t$ также "убывает" - в целых точках размерность его падает на единицу.

Очевидно, что выбор на каждом шаге симплекса $\Delta^{i} \subset \Delta^{i+1}$ неоднозначен, откуда получается древесная структура на множестве иерархий псевдоторических структур. Кроме того, максимальный симплекс $\Delta^{k}$ может быть не один; естественно определить множество всех возможных максимальных симплексов $\mathcal{S}=\left\{\Delta_{i}^{k_{i}}\right\}$, и тогда множество всех возможных иерархий псевдоторических структур представляется как конечный набор.

Комбинаторное описание выстраивающихся в иерархию псевдоторических структур также достаточно просто. Базами таких псевдоторических структур являются проективные пространства $\mathbb{C P}^{k-1}$; типы регулярных торических слоев описываются выпуклыми многогранниками, которые вырезаются из выпуклого многогранника $P_{X}$ дополнительным симплексом $\Delta^{N-k-1} \subset P_{\mathbb{C P}^{N}}$. Торический тип слоя устанавливается так: выбросив из $P_{X}$ те $k+1$ вершины, которые входят в $\Delta^{k}$, и удалив все ребра, связывающие эти вершины с оставшимися $N-k$ вершинами, мы получим новый выпуклый многогранник $P_{Y}$, который и будет соответствовать торическому слою. Таким образом, всю комбинаторику можно понять, рассматривая исходный выпуклый многогранник $P_{X}$. 
В настоящей работе также предлагается большой источник торических многообразий, выпуклые многогранники которых допускают большие симплексы $\Delta_{i}^{k_{i}}$, необходимые для построения иерархий псевдоторических структур. Идея очень простая: возьмем два гладких торических многообразия $X_{1}$ и $X_{2}$ и рассмотрим их прямое произведение $X=X_{1} \times X_{2}$. Тогда соответствующий выпуклый многогранник $P_{X}$ будет в качестве вершин иметь точки $\left\{q_{i j}\right\}$, где индекс $i$ пробегает вершины многогранника $P_{X_{1}}$, а $j$ - вершины многогранника $P_{X_{2}}$. При этом множество $E_{0}$ для $P_{X}$ будет состоять как минимум из тех ребер $\left[q_{i j}, q_{k l}\right]$ для которых $i \neq j$ и $k \neq l$. Если обозначить число вершин выпуклого многогранника как $v\left(P_{X_{i}}\right)$, то мы получим, что из $v\left(P_{X}\right)=v\left(P_{X_{1}}\right) \cdot v\left(P_{X_{2}}\right)$ вершин многогранника $P_{X}$ всегда можно выбрать $k+1=\min \left(v\left(P_{X_{1}}\right), v\left(P_{X_{2}}\right)\right)$ вершин, из которых составляется симплекс $\Delta^{k}$, необходимый для построения иерархии псевдоторических структур. Заметим при этом, что выбор $\Delta^{k}$ в этом случае далеко не единствен - на множестве таких симплексов действует прямое произведение групп перестановок вершин многогранников $P_{X_{1}}$ и $P_{X_{2}}$.

Самый простой из серии таких примеров - прямое произведение проективных пространств $\mathbb{C P}^{l} \times \mathbb{C P}^{m} ;$ при этом получаются стандартная торическая структура на поверхности дель Пеццо $\mathbb{C P}_{1}^{2}$ (случай $k=2, m=1$, максимальный симплекс - отрезок), а также псевдоторическая структура многообразия флагов $F^{3}$ из работы [3] (случай $k=m=2$, максимальный симплекс - треугольник) и ее деформация в торическую структуру на торическом вырождении многообразия флагов [3]. Нетрудно видеть, что для прямого произведения $X=\mathbb{C P}^{l} \times \mathbb{C P}^{m}$ число вершин компонент прямого произведения равно $l+1$ и $m+1$, число вершин выпуклого многогранника $P_{X}$ равно $(l+1)(m+1)$, а размерность максимального симплекса $\Delta^{k}$ есть $k=\min (l, m)$, и порог особости всегда меньше $k$ (тут дополнительно возникает зависимость от выбора последовательности вложенных симплексов). Из этого класса примеров получаем иерархии псевдоторических структур на раздутиях проективного пространства, а также на проективизациях некоторых расслоений (включая (ко)касательное) на проективных пространствах.

Псевдоторическая геометрия оказывается важным инструментом в реализации программы лагранжева геометрического квантования. Псевдоторические структуры на многообразиях позволяют строить семейства лагранжевых торов (гладких и особых, а также иных лагранжевых подмногообразий), поэтому естественно ожидать, что вместе с иерархиями псевдоторических структур возникнут иерархии семейств лагранжевых торов. В связи с тем что в лагранжевом геометрическом квантовании лагранжево подмногообразие понимается как состояние проквантованной системы, очень интересным оказывается вопрос "перерождений" лагранжевых слоев при переходе по ступеням таких иерархий. Можно ожидать, что именно в терминах таких перерождений окажется возможной реализация редукций квантовых состояний, однако ввиду важности вопроса мы откладываем обсуждение геометрии лагранжевых подмногообразий гиперплоских сечений торических многообразий до следующей работы. 


\section{2. ОСНОВНАЯ КОНСТРУКЦИЯ}

Мы будем рассматривать гладкое компактное торическое симплектическое многообразие $\left(X, \omega_{X}\right)$ вещественной размерности $2 n$ с набором коммутирующих интегралов $\left(f_{1}, \ldots, f_{n}\right)$. Комбинаторная информация о $\left(X, \omega_{X}\right)$ содержится в соответствующем выпуклом многограннике $P_{X} \subset \mathbb{R}^{n}$, и согласно теореме Дельцана многообразие $\left(X, \omega_{X}\right)$ однозначно восстанавливается из $P_{X}$. Напомним $[1]$, что $\left(X, \omega_{X}\right)$ может быть вложено в проективное пространство, $\phi: X \rightarrow \mathbb{C P}^{N}$, где $N+1$ - число целочисленных точек в $P_{X}$. При этом $\omega_{X}=\phi^{*} \omega_{\mathrm{FS}}$, где $\omega_{\mathrm{FS}}$ есть стандартная симплектическая форма метрики Фубини-Штуди на $\mathbb{C P}^{N}$, и можно выбрать набор стандартных коммутирующих морсовских функций $F_{1}, \ldots, F_{N}$ на $\mathbb{C P}^{N}$ так, что $\phi^{*} F_{i}=f_{i}$ для любого $i=1, \ldots, n$. Набор стандартных коммутирующих функций на $\mathbb{C P}^{N}$ определяется невырожденной целочисленной матрицей $A=\left(a_{i, j}\right), a_{i, j} \in \mathbb{Z}$, размера $N \times(N+1)$ :

$$
F_{i}=\frac{\sum_{j=0}^{N} a_{i, j}\left|z_{j}\right|^{2}}{\sum_{j=0}^{N}\left|z_{j}\right|^{2}}
$$

в однородных координатах $\left[z_{0}: \ldots: z_{N}\right]$. Полный набор $\left\{F_{1}, \ldots, F_{N}\right\}$ отображает все проективное пространство $\mathbb{C P}^{N}$ в $N$-мерный симплекс $P_{N} \subset \mathbb{R}^{N}$, натянутый на $N+1$ вершину $v_{0}, \ldots, v_{N}$ с координатами $v_{i} \mapsto\left(a_{1, i}, \ldots, a_{N, i}\right) \subset \mathbb{R}^{N}$.

Исходя из этого вместе с вложением $\phi$ можно построить вложение выпуклого многогранника $P_{X}$ в многогранник $P_{N}$. В самом деле, множество вершин $V\left(P_{X}\right)$ может быть естественным образом вложено в множество вершин $V\left(P_{N}\right)$ : каждая вершина многогранника $P_{X}$ есть совместная критическая точка функций $f_{1}, \ldots, f_{n}$, которой соответствует некоторая совместная критическая точка функций $F_{1}, \ldots, F_{n}$, а значит, и всех функций $F_{1}, \ldots, F_{N}$, т. е. некоторая вершина симплекса $P_{N}$. В симплексе $P_{X}$ некоторые вершины попарно соединены ребрами, а в симплексе $P_{N}$ каждая пара вершин соединена ребром, поэтому подмножество вершин $V\left(P_{X}\right) \subset V\left(P_{N}\right)$ может быть дополнено подмножеством тех ребер из $P_{N}$, которые соответствуют имеющимся ребрам в $P_{X}$. Из полноты симплекса $P_{N}$ следует, что в результате последовательного по размерностям достраивания граней многогранника $P_{X}$ мы получим вложение $P_{X}$ в $P_{N}$. Заметим, что совпадение образа вложения $P_{X}$ со всем $P_{N}$ возможно в том и только том случае, когда само $X$ является проективным пространством той же размерности, а $\phi-$ изоморфизмом. Построенный образ вложения $P_{X}$ мы будем обозначать тем же символом, если это не может привести к недоразумению.

Строгое вложение $P_{X} \subset P_{N}$ влечет следующее замечание: в то время как множество вершин $V\left(P_{X}\right)$ может совпадать с $V\left(P_{N}\right)$, множество ребер $E\left(P_{X}\right) \subset E\left(P_{N}\right)$ всегда меньше, чем множество ребер $E\left(P_{N}\right)$. В самом деле, если $E\left(P_{X}\right)=E\left(P_{N}\right)$, то вещественная размерность пространства $X$ должна равняться $2 N$, а это возможно, если и только если $X=\mathbb{C P}^{N}$ и $P_{X}=P_{N}$.

Пример 1. Рассмотрим вложения $X_{1}=\mathbb{C P}^{1}$ в $\mathbb{C P}^{2}$ по Веронезе и $X_{2}=\mathbb{C P}^{1} \times \mathbb{C P}^{1}$ в $\mathbb{C P}^{3}$ по Сегре [5]. В первом случае $P_{X_{1}}$ является одномерным симплексом, т. е. отрезком, который вкладывается в двумерный симплекс как одна сторона в треугольнике (поскольку третья вершина $P_{2}$ не принадлежит образу проективной прямой). 
Во втором случае $P_{X_{2}}$ есть прямое произведение отрезков $P_{1} \times P_{1}$ с четырьмя вершинами, которое вкладывается в тетраэдр $P_{3}$. В первом случае $V\left(P_{X_{1}}\right) \neq V\left(P_{2}\right)$, в то время как во втором случае $V\left(P_{X_{2}}\right)=V\left(P_{3}\right)$, но при этом, конечно, четыре ребра $P_{X_{2}}$ заполняют только четыре из шести ребер тетраэдра.

Рассмотрим сначала случай минимального вложения $V\left(P_{X}\right)=V\left(P_{N}\right)$. Очевидно, что в этом случае $\phi(X)$ не содержится ни в одной гиперплоскости в $\mathbb{C P}^{N}$. Рассмотрим множество подсимплексов $\left\{\Delta_{i}^{k_{i}} \subset P_{N}\right\}$ размерностей $k_{i}$, обладающих следующим свойством.

ОПРЕДЕЛЕНИЕ 1 . Подсимплекс $\Delta_{i}^{k_{i}} \subset P_{N}$, натянутый на $k_{i}+1$ вершину симплекса $P_{N}$, называется свободным, если пересечение $\Delta_{i}^{k_{i}} \cap P_{X}$ состоит только из вершин, т. е. ни одно ребро из $\Delta_{i}^{k_{i}}$ не содержится в $E\left(P_{X}\right)$. Свободный подсимплекс $\Delta_{i}^{k_{i}}$ назовем максималъным, если он не содержится ни в каком большем свободном подсимплексе.

Обозначим множество свободных подсимплексов как $\mathcal{F}$, а подможество свободных симплексов размерности $j$ - как $\mathcal{F}_{j} \subset \mathcal{F}$. Вложение $P_{X} \subset P_{N}$ строгое, следовательно, множество $\mathcal{F}$ непусто: как минимум компонента $\mathcal{F}_{1} \neq \varnothing$.

Гиперплоским сечением называется пересечение $\phi(X) \cap H \subset \phi(X)$ с гиперплоскостью $H \subset \mathbb{C P}^{N}$; множество таких пересечений при вариации гиперплоскости $H$ составляет полный линейный ряд $|H|_{X}$ - семейство дивизоров, задающихся нулями голоморфных сечений соответствующего линейного расслоения $\phi^{*} \mathcal{O}(1)$ на $X[5]$. Достаточно общее гиперплоское сечение есть гладкое подмногообразие в $\phi(X)$; нетрансверсальные пересечения приводят к возникновению особенностей, откуда можно вычислить размерность подмногообразия $\operatorname{Det}_{\mathrm{X}} \subset|\mathrm{H}|_{\mathrm{X}}$, состоящего из негладких гиперплоских сечений.

Пусть $\Delta_{i}^{k_{i}}-$ свободный подсимплекс. Без ограничения общности мы можем считать, что он натянут на первые $k_{i}+1$ вершин $v_{0}, \ldots, v_{k_{i}}$ симплекса $P_{N}$, для которых однородные координаты прообразов относительно отображения действия

$$
F_{\text {act }}=\left(F_{1}, \ldots, F_{N}\right): \mathbb{C P}^{N} \rightarrow \mathbb{R}^{N}
$$

задаются правилом $v_{j}=F_{\text {act }}\left(p_{j}\right) \mapsto[0: \ldots: 1: \ldots: 0]$, где единица стоит на $j$-м месте. В полном линейном ряду $|H|_{X}$ рассмотрим подпространство

$$
\mathbb{P}\left(\Delta_{i}^{k_{i}}\right)=\left\{\phi(X) \cap H \mid H=\sum_{j=0}^{k_{i}} \alpha_{j} z_{j}=0\right\} \subset|H|_{X},
$$

которое изоморфно проективному подпространству размерности $k_{i}$.

Пусть $M_{0}=\phi(X) \cap H_{0}$, где $H_{0} \in \mathbb{P}\left(\Delta_{i}^{k_{i}}\right)$. Тогда имеем следующее основное утверждение.

ОСНОВНАЯ ТЕОРЕмА. Пусть $n>k_{i}$. Тогда на многообразии $M_{0}$ имеется естественная псевдоторическая структура ранга $k_{i}-1$.

ДокАЗАтЕЛЬство дается явным построением. Напомним [2], что псевдоторическая структура состоит из двух типов данных - вещественного набора функций $\left(f_{1}, \ldots, f_{l}\right)$ и “комплексного" семейства симплектических подмногообразий $D_{p}$ 
размерности $2 l$, параметризуемого симплектическим торическим многообразием $Y \ni p$ дополнительной размерности. Мы начнем с построения функций $f_{i}$ и семейства $D_{p}$.

Рассмотрим глобальные функции $F_{1}, \ldots, F_{n} \in\left\{F_{1}, \ldots, F_{N}\right\}$ из полного набора коммутирующих первых интегралов на $\mathbb{C P}^{N}$, сохраняющие гамильтоновым действием наше торическое многообразие $\phi(X)$. При этом очевидно, что любая функция из линейной оболочки $\left\langle F_{1}, \ldots, F_{n}\right\rangle$ также сохраняет многообразие $\phi(X)$. Из линейной оболочки набора $F_{1}, \ldots, F_{n}$ мы хотим выделить поднабор функций $f_{i}$ таких, что каждая $f_{i}$ сохраняет гиперплоскость $H_{0}=\left\{\sum_{j=0}^{k_{i}} \alpha_{j} z_{j}=0\right\}$ своим гамильтоновым действием. Но для этого необходимо и достаточно, чтобы для каждой из таких $f_{i}$ выполнялись условия

$$
f_{i}\left(p_{0}\right)=\cdots=f_{i}\left(p_{j}\right)=f_{i}\left(p_{k_{i}}\right),
$$

из линейности которых следует, что набор существует и состоит из $l=n-k_{i}-1$ функций $f_{1}, \ldots, f_{l}$. Заметим, что функции $f_{1}, \ldots, f_{l}$ могут быть выбраны так, что $\left.f_{i}\right|_{\phi(X)}$ имеет только изолированные критические точки, поскольку $\phi(X)$ не содержит проективных прямых, натянутых на вершины $p_{0}, \ldots, p_{k_{i}}$, по определению свободного симплекса $\Delta_{i}^{k_{i}}$.

Так как каждая функция $f_{i}$ из набора $\left(f_{1}, \ldots, f_{l}\right)$ сохраняет своим гамильтоновым действием и $\phi(X)$, и $H_{0}$, это остается верным также для пересечения $M_{0}=$ $\phi(X) \cap H_{0}$. Более того, все эти функции остаются морсовскими. Этим представлен первый ингредиент псевдоторической структуры.

Рассмотрим в объемлющем проективном пространстве $\mathbb{C P}^{N}$ два проективных подпространства $L_{1}=\mathbb{C P}^{k_{i}}$ и $L_{2}=\mathbb{C P}^{N-k_{i}-1}$, определяющиеся следующими условиями: подпространство $L_{1}$ натянуто на точки $p_{0}, \ldots, p_{k_{i}}$, а $L_{2}$ - на все остальные точки $p_{k_{i}+1}, \ldots, p_{N}$, являющиеся прообразами вершин $v_{k_{i}+1}, \ldots, v_{N} \subset P_{N}$ относительно отображения действия. Заметим, что по определению $L_{2} \subset H_{0}$, но $M_{0}$ не содержится в $L_{2}$ и не содержит $L_{1}$, так как проективные прямые, натянутые на точки $p_{0}, \ldots, p_{k_{i}}$, не содержатся в $\phi(X)$. Более того, нетрудно видеть, что $M_{0} \cap L_{1}=\varnothing$. В самом деле, каждая функция из набора $F_{1}, \ldots, F_{n}$ сохраняет $\phi(X)$ и $L_{1}$, причем на $L_{1}$ гамильтоново действие функции $F_{i}$ нетривиально, за исключением стационарных точек $p_{0}, \ldots, p_{k_{i}}$, поэтому если бы $\phi(X)$ пересекалось с $L_{1}$ помимо этих точек, то $\phi(X)$ с необходимостью содержало бы всё пространство $L_{1}$, что невозможно. Отсюда следует, что $M_{0}$ не пересекается с $L_{1}$.

Рассмотрим семейство проективных пространств $\widetilde{D}_{t}$, параметризованное точками $t \in L_{1} \cap H_{0}$, так что $\widetilde{D}_{t}$ есть пересечение $H_{0}$ с проективной оболочкой $L_{2}$. Также рассмотрим точки $t \in L_{1}$ такие, что

$$
\widetilde{D}_{t}=\left\langle L_{2}, t\right\rangle \cap H_{0}, \quad t \in L_{1} \cap H_{0}, \quad \operatorname{dim} \widetilde{D}_{t}=N-k_{i}-1 .
$$

Нетрудно видеть, что для любой точки $t \in L_{1} \cap H_{0}$ пересечение $\widetilde{D}_{t} \cap M_{0} \subset H_{0}$ непусто: это следует из условия теоремы, поскольку, если $n>k_{i}$, размерность многообразия $M_{0}$ (равная $\left.n-1\right)$ оказывается не меньше коразмерности пространства $\widetilde{D}_{t} \subset H_{0}$ (равной $k_{i}$ ).

Возьмем в качестве семейства подмногообразий $M_{0}$ множество пересечений

$$
D_{t}=\widetilde{D}_{t} \cap M_{0} \subset M_{0}, \quad t \in L_{1} \cap H_{0} .
$$


Покажем, что каждое подмногообразие $D_{t}$ имеет одну и ту же размерность, равную $2\left(n-k_{i}-1\right)$. Для этого покажем, что первое условие согласования вещественных и комплексных данных в нашей конструкции имеет место, а именно, что все функции из набора $\left(f_{1}, \ldots, f_{l}\right)$ сохраняют своим гамильтоновым действием каждое подмногообразие $D_{t}$ для любого $t \in Y=L_{1} \cap H_{0}$.

В самом деле, по построению функции $f_{i}$ сохраняют $M_{0}$ и $L_{2}$, а на $L_{1}$ ограничиваются константами. Отсюда следует, что каждое подмногообразие $\widetilde{D}_{t}$ также сохраняется гамильтоновым действием любой из $f_{i}$. Но отсюда следует, что пересечение $D_{t}=M_{0} \cap \widetilde{D}_{t}$ также сохраняется гамильтоновым действием любой из $f_{i}$. Кроме того, имеется однородное действие на самой гиперплоскости $H_{0}$, перемещающее слои многообразия $\widetilde{D}_{t}$, откуда следует равенство размерностей слоев. Но если слои имеют одну размерность, то они все имеют одну и ту же - трансверсальную - размерность. Следовательно, в общей точке пространство, касательное к $D_{t}$, должно порождаться гамильтоновыми векторными полями $X_{f_{i}}$, и $\left(D_{t}, f_{1}, \ldots, f_{l}\right)$ представляют фазовые пространства вполне интегрируемых систем.

Для завершения доказательства нам остается проверить "сложное" условие согласования симплектических форм на $M_{0}$ и $Y$. Напомним [2], что́ это условие означает в нашем случае. Рассмотрим комплексное отображение

$$
\psi: M_{0} \backslash B \rightarrow Y
$$

компактификация слоев которого дает элементы семейства $\left\{D_{t}\right\}$, а именно

$$
D_{t}=\overline{\psi^{-1}(t)}=\psi^{-1}(t) \cup B, \quad t \in Y,
$$

где базисное множество $B$ есть просто $L_{2} \cap M_{0} \subset M_{0}$ (выше мы показали, что включение строгое). В нашем случае $\psi$ является проекцией в проективном пространстве $H_{0}$ многообразия $M_{0}$ из $L_{2}$ в $L_{1} \cap H_{0}$ (о проекциях в проективном пространстве см. монографию [5]). Заметим при этом, что симплектические формы на $M_{0}$ и $Y$ получаются ограничением на эти подмногообразия стандартной формы $\omega_{\mathrm{FS}}$ кэлеровой метрики Фубини-Штуди на $\mathbb{C P}^{N}$. "Сложное" условие согласования из определения псевдоторической структуры выглядит так: для любой гладкой вещественной функции $h$ на $Y$ имеет место тождество

$$
X_{\psi^{*} h} \wedge \nabla_{\psi} X_{h}=0 \quad \text { на } \quad M_{0} \backslash B
$$

где гамильтоновы векторные поля $X_{\psi^{*}}$ и $X_{h}$ определены на $M_{0}$ и $Y$ соответственно, a $\nabla_{\psi}$ - естественная связность на расслоении с симплектическими слоями [2].

Проверим тождество (1) сначала для проекции $\tilde{\psi}: H_{0} \backslash L_{1} \rightarrow H_{0} \cap L_{1}=Y$ всей гиперплоскости $H_{0}$ (напомним, что $L_{2} \subset H_{0}$ ). Произвольная гладкая функция на $Y$ может быть записана как функция $h\left(z_{1}, \ldots, z_{k_{i}}, \bar{z}_{1}, \ldots, \bar{z}_{k_{i}}\right)$, так как переменную $z_{0}$ можно исключить $\left(H_{0}\right.$ - достаточно общая гиперплоскость, поэтому первый коэффициент $\alpha_{0}$ в уравнении для нее можно считать не равным нулю), и подьем $\tilde{\psi}^{*} h$ на $H_{0}$ можно записать ровно в том же виде (поскольку подъем не зависит от всех остальных координат). Так как форма $\omega_{\mathrm{FS}}$ в каждой точке пропорциональна $\sum_{i=0}^{N} d z_{i} \wedge d \bar{z}_{i}$, а ее ограничение на $Y$ пропорционально $\sum_{i=0}^{k_{i}} d z_{i} \wedge d \bar{z}_{i}$, гамильтоновы векторные поля $X_{\tilde{\psi}^{*} h}$ и $\nabla_{\tilde{\psi}} X_{h}$ пропорциональны друг другу в каждой точке из $H_{0} \backslash L_{2}$, что равносильно условию (1). 
Далее, чтобы ограничить это утверждение на $M_{0}$ и $\psi$, достаточно заметить, что в точках $M_{0} \subset H_{0}$ симплектический ортогонал к слою $\psi$ совпадает с симплектическим ортогоналом к слою $\tilde{\psi}$, откуда видно, что $X_{\tilde{\psi}^{*} h}$ принадлежит касательному пространству $T M_{0} \subset T H_{0}$, и мы имеем равенство $X_{\tilde{\psi}^{*} h}=X_{\psi^{*} h}$ в точках множества $M_{0} \backslash B$ и одновременно совпадение связностей $\nabla_{\tilde{\psi}}$ и $\nabla_{\psi}$. Таким образом, тождество (1) верно и для отображения $\psi: M_{0} \backslash B \rightarrow Y$, что завершает доказательство теоремы.

Пример 2. В работе [3] была построена псевдоторическая структура на многообразии флагов $F^{3}$, которая оказывается структурой именно такого типа, который получен в основной теореме. В самом деле, в качестве исходного торического многообразия $X$ рассмотрим прямое произведение $\mathbb{C P}^{2} \times \mathbb{C P}^{2}$ и вложим его по Веронезе в $\mathbb{C P}^{8}$. Тогда выпуклый многогранник $P_{X}$ имеет девять вершин $v_{i, j}, i, j=0,1,2$, и шесть ребер, каждое из них связывает вершины, у которых один из индексов совпадает. Таким образом, у нас имеются три вершины $v_{0,0}, v_{1,1}, v_{2,2}$, не соединенные ребрами, и в качестве свободного симплекса $\Delta^{2} \subset P_{8}$ полного симплекса с девятью вершинами можно взять треугольник с вершинами $v_{i, i}$. Тогда в качестве гиперплоскости $H_{0}$ можно взять гиперплоскость, которая задана уравнением, соответствующим условию $\sum_{i=0}^{2} x_{i} y_{i}=0$ (см. статью [3]), что дает многообразие флагов. Нетрудно проверить, что мы получим в точности ту же псевдоторическую структуру.

Таким образом, основная конструкция настоящей работы максимально обобщает конструкцию псевдоторической структуры на многообразии флагов из работы [3].

\section{3. КОМБИНАТОРИКА}

Обсудим комбинаторные аспекты возникновения псевдоторических структур на гиперплоских сечениях торических многообразий. Так как торическое многообразие однозначно задается своим выпуклым многогранником, задача будет состоять в том, как по выпуклому многограннику исходного торического многообразия $X$ найти выпуклые многогранники торических слоев псевдоторических структур, возникающих в результате применения основной теоремы.

Начнем со следующего простого замечания. Пусть $X$ - гладкое торическое многообразие и $P_{X}$ - соответствующий ему выпуклый многогранник. Тогда две существенные характеристики многообразия $X$ - это число вершин $v=\left|V\left(P_{X}\right)\right|$ и валентность вершин $w$ (напомним, что для гладкого торического многообразия валентность всех вершин одинакова и равна комплексной размерности многообразия $X$ ). Тогда если $X \subset \mathbb{C P}^{N}$ - минимальное вложение, то $N=v-1$ и $\operatorname{codim} X=v-1-w$. Если $\Delta^{k} \subset \Delta^{N}-$ свободный симплекс, то имеется следующее необходимое условие на $k$.

УтВЕРЖДЕНИЕ 1. В случае минимального вложения $k \leqslant \operatorname{codim} X$.

ДокАЗАтельство. Общее число вершин в $\Delta^{N}$ равно $N+1$, так что одну вершину симплекса $\Delta^{k}$ нельзя связать более чем с $N-w$ вершинами, которые бы не были связаны ребрами из $P_{X}$. Этим доказано искомое неравенство.

С другой стороны, утверждение 1 доставляет необходимое, но далеко не достаточное условие существования свободного симплекса. Можно только точно указать 
на то, что если $\operatorname{codim} X>0$, то существует свободный симплекс $\Delta^{1}$. В самом деле, если валентность вершин многогранника $P_{X}$ меньше их числа, то для каждой вершины найдется такая вершина, которая не соединена с первой ребром, поэтому пара этих вершин может быть взята в качестве вершин свободного симплекса $\Delta^{1}$. Основная теорема в этом случае обеспечивает существование торической структуры на (скорее всего) негладком гиперплоском сечении многообразия $X$.

ПримеР 3. Нетрудно построить пример, когда $k$ не достигает верхней границы $\operatorname{codim} X$. Пусть $X$ имеет размерность 3 , т. е. $w=3$, тогда число вершин обязано быть четным, и, пропуская первый случай $v=4$ (когда $P_{X}$ есть $\Delta^{3}$ ), рассмотрим случай $v=6$. Тогда $P_{X}$ имеет девять ребер и пять граней, т. е. является треугольной призмой. Несмотря на то что в этом случае $v-1-w=2$, не существует свободного симплекса $\Delta^{2}$. Заметим, что по виду $P_{X}$ легко восстанавливается $X$ как прямое произведение $\mathbb{C P}^{2} \times \mathbb{C P}^{1}$, вложенное в $\mathbb{C P}^{5}$, так что основная теорема доставляет в этом случае торическую стандартную структуру на гиперплоском сечении многообразия $X$, изоморфном раздутию проективной плоскости в одной точке [5].

Таким образом, для нахождения интересных примеров псевдоторических многообразий нам необходимо представить набор таких торических многообразий, в выпуклых многогранниках которых есть место для свободных симплексов размерности большей или равной двум. В настоящей работе представлены два источника таких торических многообразий.

Прямое произведение. Рассмотрим пару симплектических компактных торических многообразий $X_{1}, X_{2}$; тогда их прямое произведение $X=X_{1} \times X_{2}$ снова будет торическим. Соответствующий выпуклый многогранник $P_{X}$ будет вкладываться в выпуклый многогранник $P_{\text {univ }}$, получающийся следующим образом: берем все пары вершин $v_{i, j}=\left(v_{i}, v_{j}\right) \in V\left(P_{X_{1}}\right) \times V\left(P_{X_{2}}\right)$, где $V\left(P_{X_{i}}\right)$ обозначает множество вершин выпуклого многогранника, соответствующего торическому многообразию $X_{i}$, и строим выпуклый симплекс с вершинами $v_{i, j}$. Очевидно, что это будет соответствовать минимальному вложению торического многообразия $X$ в проективное пространство; при этом для описания возникающей псевдоторической структуры нам не обязательно, чтобы существовало такое минимальное вложение. В самом деле, пусть вложение $X \subset \mathbb{C P}^{N}$ не минимально. Тогда свободный симплекс, использовавшийся в предыдущем разделе для построения псевдоторической структуры, может включаться в больший по размерности свободный симплекс в $\Delta^{N+1}$, однако для построения псевдоторической структуры достаточно брать меньший из двух. Выбор этого свободного симплекса универсален - он определен вложением $P_{X} \subset P_{\text {univ }}$, поэтому нам не надо искать настоящее вложение в проективное пространство для комбинаторного описания.

Итак, комбинаторная задача такова: по двум выпуклым многогранникам, соответствующим торическим многообразиям $X_{1}, X_{2}$, описать максимальные свободные симплексы в многограннике $P_{\text {univ }}$ и многогранники соответствующих (возможно, особых) торических слоев. Имеем следующее

УтВеРЖДЕНИЕ 2. Максималъный свободный симплекс $\Delta^{k} \subset P_{\text {univ }}$ имеет не менее чем $\min \left(\left|V\left(P_{X_{1}}\right)\right|,\left|V\left(P_{X_{2}}\right)\right|\right)$ вершин, где $\left|V\left(P_{X_{i}}\right)\right|$ - число вершин в $P_{X_{i}}$. 
ДоказАтельство. Вершины $v_{i, j}$ и $v_{k, l}$ в $P_{\text {univ }}$ могут быть связаны ребром, если $\delta_{k}^{i}+\delta_{l}^{j}=1$ (если эта сумма равна 2, то вершина одна и та же). Это условие достаточное, откуда следует необходимое утверждение.

Из утверждения 2 вытекает существование свободного симплекса для любой пары $X_{1}, X_{2}$ торических многообразий. Нетрудно видеть, что такой универсальный свободный симплекс $\Delta_{\text {univ }}$ задается выбором нумераций вершин многогранника $P_{X_{i}}$. В самом деле, выберем произвольные нумерации на множествах $V\left(P_{X_{i}}\right)$; тогда каждая пара вершин из набора $\left\{v_{i, i}\right\}$ не соединена ребром, что влечет существование свободного симплекса с вершинами в этом наборе. Отложив вопрос о максимальности такого свободного симплекса, проработаем в максимальной общности вопрос о комбинаторной структуре торических слоев псевдоторической структуры, существование которой обеспечивается основной теоремой. Имеем следующее

УТВЕРЖДЕНИЕ 3. Общий комбинаторный тип торического слоя псевдоторической структурь, конструкция которой изложена в основной теореме, определяется выпуклым многогранником $P_{0} \subset P_{\text {univ }}$, получающимся удалением из $P_{\text {univ }}$ всех вершин универсального симплекса $\Delta_{\text {univ }}$.

ДокАЗАТЕЛЬСтво. По построению каждый слой является (возможно, особым) торическим многообразием, снабженным набором первых интегралов с общими критическими точками в точности в тех вершинах $P_{\text {univ }}$, которые остаются после удаления вершин симплекса $\Delta_{\text {univ }}$. Множества вырождения набора гамильтоновых векторных полей этих интегралов суть всё те же проективные оболочки оставшихся в $P_{0}$ вершин, на которых эти наборы вырождались в объемлющем проективном пространстве. Это следует из того, что все эти гамильтоновы векторные поля касаются торических слоев псевдоторической структуры. Таким образом, при отображении моментов, задающемся этими первыми интегралами, образом торического слоя будет выпуклый многогранник $P_{0}$, что и требовалось доказать.

Пример 4. Рассмотрим прямое произведение $\mathbb{C P}^{k} \times \mathbb{C P}^{l}, k \leqslant l$. В этом случае нижняя граница, определяющаяся утверждением 2 , является одновременно и верхней. В самом деле, в этом случае выпуклые многогранники $P_{X_{i}}$ являются симплексами, в которых каждая пара вершин соединена ребром, поэтому единственная возможность получить свободный симплекс - перенумеровать вершины в $P_{X_{i}}$ произвольным образом и натянуть симплекс $\Delta^{k}$ на вершины $\left\{v_{i, i}, i=1, \ldots, k+1\right\}$. Тогда согласно основной теореме существует псевдоторическая структура на гиперплоском сечении $X$ комплексной размерности $k+l-1$ с базой $\mathbb{C} \mathbb{P}^{k-1}$, общий торический слой которой имеет размерность $k+l-1-(k-1)=l$. Заметим, что после удаления $\Delta_{\text {univ }}$ из $P_{\text {univ }}$ мы получаем выпуклый многогранник $P_{0}$, число вершин которого равно $(k+1) l$. Вычислим валентность вершин многогранника $P_{0}$. Вершина $v_{i, j} \subset P_{0}$, $i \neq j$, до удаления $\Delta_{\text {univ }}$ была связана с $k+l$ вершиной многогранника $P_{\text {univ }}$. В peзультате вырезания $\Delta_{\text {univ }}$ из числа этих вершин исключаются ровно две вершины, а именно $v_{i, i}$ и $v_{j, j}$, если $j \leqslant k+1$, или ровно одна вершина $v_{i, i}$ если $j>k+1$. То есть в случае $k \neq l$ вершины многогранника $P_{0}$ имеют разную валентность, откуда следует, что общий слой соответствующей псевдоторической структуры на гиперплоском сечении $\mathbb{C P}^{k} \times \mathbb{C P}^{l}$ является негладким. Однако такая псевдоторическая 
структура будет регулярной, потому что особенности ее слоев будут содержаться в базисном множестве.

Пусть теперь $k=l$, найдем в этом случае условие того, что псевдоторическая структура, доставляемая основной теоремой, будет иметь гладкие торические слои. Как уже было сказано, размерность торического слоя в этом случае равна $l=k$, а валентность вершин многогранника $P_{0}$ равна $k+l-2=2 k-2$. Гладкое торическое многообразие имеет ту же размерность, какова валентность вершин соответствующего выпуклого многогранника [1], откуда $k=2 k-2$, т. е. $k=2$. В точности этот случай - псевдоторической структуры на многообразии флагов $F^{3}$ - представлен в работе [3]. Оказывается, других подобных примеров с гладкими торическими слоями не существует. Но информация о негладких слоях тоже бывает полезна. Рассмотрим случай $k=3$, т. е. гиперплоское сечение прямого произведения $\mathbb{C P}^{3} \times \mathbb{C P}^{3}$. Торический слой имеет размерность 3 , число вершин $P_{0}$ равно $16-4=12$, валентность вершин равна 4. Так как размерность не совпадает с валентностью, торический слой негладкий - имеются 12 особых точек, соответствующих вершинам $P_{0}$.

ПримеР 5. Известно [1], что проективная плоскость $X=\mathbb{C P}_{1}^{2}$ с одной раздутой точкой, будучи торическим многообразием, соответствует выпуклому четырехугольнику $P_{X} \subset \mathbb{R}^{2}$. Рассмотрим гиперплоское сечение прямого произведения $X \times \mathbb{C P}^{1}$. Выпуклый многогранник прямого произведения $X \times \mathbb{C P}^{1}$ представляет собой четырехугольную призму $P_{X} \times I$ с вершинами $\left\{v_{i, 1}, v_{i, 2}\right\}$, где $i=1, \ldots, 4$. Выберем нумерацию вершин в $P_{X}$ так, что вершины соединены ребром в $P_{X}$, если и только если их четность противоположна. Тогда в призме $P_{X} \times I$ мы имеем свободный симплекс $\Delta^{2}$ с вершинами $v_{1,1}, v_{2,2}, v_{3,1}$. Гиперплоское сечение $X \times \mathbb{C P}^{1}$ есть проективная плоскость $\mathbb{C P}_{2}^{2}$ с двумя раздутыми точками [5], которая также является торическим многообразием, и торическая структура может быть восстановлена с помощью основной теоремы, если в качестве свободного симплекса взять $\Delta^{1}$ с вершинами $v_{1,1}$, $v_{2,2}$. Однако выбор большего свободного симплекса $\Delta^{2}$ приводит нас к псевдоторической структуре ранга 1 на $\mathbb{C P}_{2}^{2}$; комбинаторное описание торического слоя этой структуры дается вычеркиванием трех вершин симплекса $\Delta^{2}$ из $P_{X} \times I$. При этом мы получаем несвязный граф, который соответствует трем неподвижным базисным компонентам и еще одной базисной точке, соответствующей невырожденному максимуму единственного первого интеграла, который сохраняет слои получающейся псевдоторической структуры.

Другой путь, ведущий к нетривиальным свободным симплексам, предполагает обещанную в предыдущем разделе модификацию основной теоремы на случай неминимального вложения $X \subset \mathbb{C P}^{N}$. Однако, как мы увидим ниже, в этом случае свободных симплексов оказывается слишком много, и нам придется принудительно вводить на их размерность некоторые ограничения.

Неминимальное вложение. Выше мы доказали основную теорему в случае минимального вложения $X \subset \mathbb{C P}^{N}$, т. е. когда множество вершин многогранника $P_{X}$ равно $N+1$. Однако в общем случае число вершин симплекса $\Delta^{N}$ может оказаться больше числа вершин многогранника $P_{X}$. Теорема может быть модифицирована и на этот случай со следующими уточняющими деталями.

Прежде всего, существование в $\Delta^{N}$ не только свободных ребер, но и свободных вершин существенно расширяет множество потенциальных свободных симплексов. 
В самом деле, пусть вершина $v_{0} \in P_{X} \subset \Delta^{N}$ произвольным образом выбрана из множества вершин $P_{X}$, а вершины $v_{1}, \ldots, v_{k} \in \Delta^{N}$ не содержатся в $P_{X}$. Тогда симплекс $\Delta^{k}$, натянутый на вершины $v_{0}, \ldots, v_{k}$, обладает тем же свойством, что и свободный симплекс, поэтому мы можем применить основную теорему. Однако в минимальном случае мы имели следующие ограничения на размерности:

- размерность самого́ торического многообразия $X$ равна валентности вершин многогранника $P_{X}$;

- размерность $k$ свободного симплекса $\Delta^{k}$ не может быть больше разности валентностей вершин $\Delta^{N}$ и $P_{X}$,

$$
k \leqslant N-\operatorname{dim} X=\operatorname{codim} X
$$

- размерности гиперплоского сечения $H \cap X$, базы и слоя псевдоторической структуры на $H \cap X$ равны соответственно $\operatorname{dim} X-1, k-1$ и $\operatorname{dim} X-k$.

Слой псевдоторической структуры должен быть торическим многообразием; его набор первых интегралов выделен из набора первых интегралов $F_{1}, \ldots, F_{\operatorname{dim} X}$ самого́ многообразия $X$ несколькими линейными соотношениями, которые налагаются условиями сохранения гиперплоскости $H$. Если гиперплоскость $H$ в $\mathbb{C P}^{N}$ проходит ровно через $d \leqslant N-1$ вершин симплекса $\Delta^{N}$, то ей соответствуют ровно $N-d-1$ линейных условий; следовательно, если $H$ не проходит в точности через те вершины симплекса $\Delta^{N}$, которые принадлежат свободному симплексу $\Delta^{k}$, то это соответствует наложению ровно $k$ линейных условий на набор $F_{1}, \ldots, F_{\operatorname{dim}} X$ первых интегралов. Отсюда следует совпадение размерностей слоев псевдоторической структуры с числом первых интегралов, сохраняющих эти слои.

Перейдем теперь к неминимальному случаю. На этот раз имеются не только свободные ребра, но и свободные вершины в $\Delta^{N}$, но если эти вершины использовать при построении свободного симплекса, то получится слишком большая размерность такого свободного симплекса. Например, пусть вершины $v_{1}, \ldots, v_{k} \in \Delta^{N}$ не принадлежат $P_{X}$, а некоторая вершина $v_{0} \in P_{X} \subset \Delta^{N}$. Тогда формально симплекс $\Delta^{k}$, натянутый на вершины $v_{0}, \ldots, v_{k}$, будет свободным, и можно применить основную теорему. Однако, так как гиперплоскость $H$ по построению не содержит вершин $v_{0}, \ldots, v_{k} \subset \Delta^{N}$, число первых интегралов $F_{1}, \ldots, F_{\operatorname{dim} X}$ уменьшается на $k$, и поэтому число первых интегралов, сохраняющих гиперплоское сечение $H \cap X$, может оказаться равным нулю; в этом случае псевдоторическая структура не может существовать. Отсюда мы имеем первое ограничение.

УтВЕРЖДЕНИЕ 4. Число первъх интегралов, сохраняющих гиперплоское сечение $X \cap H$, равно $\operatorname{dim} X-k$ в случае общей гиперплоскости $H$, не проходящей через $k+1$ mочек $v_{0}, \ldots, v_{k}$.

ДокАзАтЕЛЬство. Уравнение общей гиперплоскости $H$, не проходящей через вершины $v_{0}, \ldots, v_{k}$, имеет вид $\sum_{i=0}^{k} \alpha_{i} z_{i}=0$; здесь вершина $v_{i}$ задана однородными координатами $[0: \ldots: 0: 1: 0: \ldots: 0]$, где единица стоит на $i$-м месте. Таким образом, если первый интеграл

$$
F_{i}=\frac{\sum_{j=0}^{N} \lambda_{j}^{i}\left|z_{j}\right|^{2}}{\sum_{j=0}^{N}\left|z_{j}\right|^{2}},
$$


задающийся набором критических значений $\left(\lambda_{0}^{i}, \ldots, \lambda_{N}^{i}\right)$, сохраняет торическое многообразие $X \subset \mathbb{C P}^{N}$, то гиперплоское сечение $H \cap X$ сохраняется интегралом $F_{i}$, если и только если $\lambda_{0}^{i}=\cdots=\lambda_{k}^{i}$, что дает ровно $k$ линейных соотношений на критические значения. Поскольку исходно мы можем полагать, что критические значения различны (это эквивалентно тому, что все $F_{i}$ - морсовские функции) в случае гладкого торического многообразия $X$, наложение этих $k$ линейных условий выделяет в наборе $\left(F_{1}, \ldots, F_{\operatorname{dim} X}\right)$, определенном с точностью до действия группы $G L(\operatorname{dim} X, \mathbb{R})$, поднабор $\left(F_{1}^{\prime}, \ldots, F_{\operatorname{dim}}^{\prime} X-k\right)$ первых интегралов, сохраняющих $H \cap X$. При этом уже нельзя утверждать, что все функции $F_{j}^{\prime}$ окажутся морсовскими на пересечении $H \cap X$. Утверждение доказано.

Таким образом, на пересечении $H \cap X$ имеются $\operatorname{dim} X-k$ первых интегралов, т. е. в точности столько, какова размерность торических слоев псевдоторической структуры. Вычислим теперь ту же размерность при прямом применении основной теоремы. В проективном пространстве $H$ размерности $N-1$ имеем базовое множество $\mathbb{C P}^{k} \cap H=H_{1}-$ проективное подпространство размерности $k-1$ (здесь $\mathbb{C P}^{k}$ соответствует симплексу $\Delta^{k}$ ). Дополнительное пространство (натянутое на остальные вершины симплекса $\Delta^{N}$, которых ровно $\left.N+1-(k+1)=N-k\right) H_{2} \subset H$ имеет размерность $N-k-1$; выбор произвольной точки $p \in H_{1}$ дает проективную оболочку $H_{p}=\left\langle p, H_{2}\right\rangle \subset H$ размерности $N-k$. Отсюда следует, что пересечение $H_{p} \cap(H \cap X)$ в трансверсальном случае имеет размерность $N-k+\operatorname{dim} X-1-(N-1)=\operatorname{dim} X-k$. Для общей точки $p \in H_{1}$ пересечение имеет именно такую размерность. Но пересечение $H_{p} \cap(X \cap H)$ инвариантно относительно гамильтонова действия набора первых интегралов, поэтому касательное пространство натянуто на гамильтоновы векторные поля интегралов $F_{1}^{\prime}, \ldots, F_{\operatorname{dim} X-k}^{\prime}$. В то же время остальные первые интегралы из набора $F_{1}, \ldots, F_{\operatorname{dim} X}$ сдвигают пересечение $H_{p} \cap(X \cap H)$ внутри $X \cap H$, откуда следует, что нормальные деформации имеют (комплексную) размерность $k$, т. е. пересечение обязано иметь (комплексную) размерность $\operatorname{dim} X-k$, что и требовалось.

Отсюда видно, что основная теорема работает и в неминимальном случае по модулю следующего уточнения: мы ограничены в выборе свободного симплекса $\Delta^{k}$ требованием положительности числа $\operatorname{dim} X-k$, т. е. $k<\operatorname{dim} X$.

Пример 6. Неминимальное вложение возникает в следующей простейшей ситуации: если само $X$ является проективным пространством, то минимальное вложение не допускает существования никакого свободного симплекса, так как $P_{X}$ сам является симплексом $\Delta^{n}$. Но в этом случае можно рассмотреть вложение Сегре пространства $X$ в проективное пространство большей размерности, и это вложение, конечно, не может быть минимальным.

Рассмотрим случай $X=\mathbb{C P}^{3}$, вложим $X$ в $\mathbb{C P}^{9}$ с помощью стандартной конструкции Сегре (см., например, монографию [5]). Если $\left[z_{0}: z_{1}: z_{2}: z_{3}\right]$ - однородные координаты на $X$, то рассмотрим проективное пространство с однородными координатами $\left[z_{0}^{2}: z_{1}^{2}: z_{2}^{2}: z_{3}^{2}: \ldots: z_{i} z_{j}: \ldots\right]$, где первые четыре координаты соответствуют квадратам координат $z_{i}$, а остальные шесть - попарным произведениям $z_{i} z_{j}, i \neq j$. Очевидно, что образ $X \subset \mathbb{C P}^{9}$ (который мы ради экономии места также обозначаем как $X$ ) в качестве $P_{X} \subset \Delta^{9}$ имеет 3-симплекс, натянутый на первые четыре вершины симплекса $\Delta^{9}$ (те, для которых $z_{k}^{2}=1$, а все остальные квадраты и произведения равны нулю). 
Свободные симплексы в $\Delta^{9}$ имеются в большом количестве, но в этом неминимальном случае мы связаны соотношением $k<\operatorname{dim} X=3$. Другими словами, $k=1$ или $k=2$. Но в первом случае мы получим торическое подмногообразие в $\mathbb{C P}^{3}$, а именно невырожденную квадрику (вложение Сегре имеет степень 2), так что нетривиальная псевдоторическая геометрия начинается с $k=2$. Следовательно, мы можем выбрать три вершины в качестве вершин свободного симплекса $\Delta^{2}$. Выберем следующие вершины: пусть первая есть одна из вершин симплекса $P_{X} \subset \Delta^{9}$, а две других мы выберем так, чтобы пересечение $X \cap H$, где $H$ - общая гиперплоскость, не проходящая через эти три вершины, было гладким. Например, если взять вершины, соответствующие координатам $z_{0}^{2}, z_{1} z_{2}, z_{0} z_{0}$, то пересечением будет гладкая квадрика $Q=\left\{z_{0}^{2}=\alpha_{1} z_{1} z_{2}+\alpha_{2} z_{0} z_{3}\right\} \subset \mathbb{C P}^{3}$, где $\alpha_{i}-$ произвольные ненулевые числа. Получающаяся при этом псевдоторическая структура уже была построена в работе [2].

В следующем примере используется комбинация двух приемов, представленных выше, - взятия прямого произведения и повышения степени - для построения новой псевдоторической структуры на комплексной двумерной квадрике.

Пример 7. Рассмотрим в прямом произведении $\mathbb{C P}^{2} \times \mathbb{C P}^{1}$ с однородными координатами $\left[x_{0}: x_{1}: x_{2}\right],\left[y_{0}: y_{1}\right]$ гиперповерхность $X \subset \mathbb{C P}^{2} \times \mathbb{C P}^{1}$, задающуюся уравнением $X=\left\{x_{0} y_{0}^{2}+x_{1} y_{0} y_{1}+x_{2} y_{1}^{2}=0\right\}$. Взаимно однозначное соответствие двумерной комплексной квадрике строится следующим образом: введем однородные координаты $\left[\alpha_{0}: \alpha_{1}\right],\left[\beta_{0}: \beta_{1}\right]$ на двух копиях проективной прямой $\mathbb{C P}_{\alpha}^{1}, \mathbb{C P}_{\beta}^{1}$ и сопоставим точке прямого произведения точку многообразия $X$ по правилам

$$
x_{0}=\alpha_{0} \beta_{1}, \quad x_{1}=-\alpha_{0} \beta_{0}-\alpha_{1} \beta_{1}, \quad x_{2}=\alpha_{1} \beta_{0}, \quad y_{i}=\beta_{i} .
$$

Остается напомнить, что прямое произведение проективных прямых изоморфно двумерной квадрике [5].

Построим естественную псевдоторическую структуру на $X$, пользуясь в качестве образца построением псевдоторической структуры на многообразии флагов из работы [3]. Зададим сначала отображение

$$
\psi_{0}: \mathbb{C P}^{2} \times \mathbb{P}^{1} \backslash B \rightarrow \mathbb{C P}^{2}=\mathbb{P}\left(\mathbb{C}\left\langle w_{0}, w_{1}, w_{2}\right\rangle\right)
$$

формулами $w_{0}=x_{0} y_{0}^{2}, w_{1}=x_{1} y_{0} y_{1}, w_{2}=x_{2} y_{1}^{2}$, где базисное множество $B$ определяется условием одновременного обращения в нуль всех трех мономов, т. е. двумя рациональными кривыми $C_{N}=\left\{y_{0}=x_{2}=0\right\}$ и $C_{S}=\left\{y_{1}=x_{0}\right\}$. Обе кривые лежат на $X$, поэтому ограничение

$$
\psi: X \backslash B \rightarrow L=\left\{w_{0}+w_{1}+w_{2}=0\right\} \subset \mathbb{C P}^{2}
$$

корректно определено. Слои отображения $\psi_{0}$ инвариантны относительно гамильтонова действия функции

$$
F=\frac{2\left|x_{0}\right|^{2}-2\left|x_{2}\right|^{2}}{\sum_{i=0}^{2}\left|x_{i}\right|^{2}}+\frac{\left|y_{1}\right|^{2}-\left|y_{0}\right|^{2}}{\left|y_{0}\right|^{2}+\left|y_{1}\right|^{2}}
$$


В самом деле, торическое действие потока, задаваемого гамильтоновым векторным полем $F$, имеет веса на координатах $\left[x_{0}: x_{1}: x_{2}\right]$ и $\left[y_{0}: y_{1}\right]$, равные соответственно $(2 ; 0 ;-2)$ и $(-1 ; 1)$, откуда видно, что на мономах $x_{0} y_{0}^{2}, x_{1} y_{0} y_{1}$ и $x_{2} y_{0}^{2}$ это действие тривиально. Отсюда следует, что ограничение $f=\left.F\right|_{X}$ является подходящим кандидатом на вещественное данное псевдоторической структуры, а $\psi$ соответствует комплексным данным (в терминах определения из работы [2]).Таким образом, $(\psi, f)$ определяют псевдоторическую структуру на $X$.

Заметим, что функция $f$ является морсовской на $X$ : у нее ровно четыре изолированные критические точки с критическими значениями $(3 ; 1 ;-1 ; 3)$. Заметим, что образ базисного множества $B$ при отображении $f$ представляет собой два отрезка $[1,3]$ и $[-3,-1]$, причем первый отрезок есть образ $f\left(C_{N}\right)$, а второй - образ $f\left(C_{S}\right)$. Отрезая базисное множество $B$ от произвольного слоя $\bar{\psi}^{-1}(p), p \in L$, получаем проколотую рациональную кривую $C_{p} \subset X$ (т. е. кривую без двух точек пересечения с кривыми $C_{N}$ и $C_{S}$ ). Нетрудно видеть, что $C_{p}$ отображается функцией $f$ в интервал $(-1,1)$.

Далее, воспользуемся тем, что поверхность $X$ является двулистным накрытием пространства $\mathbb{C P}^{2}$, разветвленным в кривой второй степени $Q_{0}$. В самом деле, если рассмотреть первое прямое слагаемое в $\mathbb{C P}^{2}$ с координатами $x_{i}$, то каждой точке с координатами $\left[x_{0}: x_{1}: x_{2}\right]$ соответствует квадратное уравнение на $y_{0}, y_{1}$, которое в общем случае имеет два решения, совпадающие в том и только том случае, когда однородные координаты $\left[x_{0}: x_{1}: x_{2}\right]$ удовлетворяют квадратичному соотношению (дискриминант равен нулю). Обозначим соответствующую проекцию как

$$
\pi: X \rightarrow \mathbb{C P}_{x}^{2} \supset Q_{0}
$$

тогда нетрудно видеть, что слои отображения $\psi$ опускаются посредством этой проекции в слои отображения

$$
\psi_{0}: \mathbb{C P}_{x}^{2} \rightarrow L
$$

определенные условиями $w_{0} w_{2} x_{1}^{2}=w_{1}^{2} x_{0} x_{2}$. Если мы добавим сюда функцию

$$
F_{0}=\frac{2\left|x_{0}\right|^{2}-2\left|x_{2}\right|^{2}}{\sum_{i=0}^{2}\left|x_{i}\right|^{2}},
$$

то мы получим хорошо знакомую нам из работ [2], [6] псевдоторическую структуру на $\mathbb{C P}_{x}^{2}$. Заметим, что кривая ветвления $Q_{0}$ сама является слоем псевдоторической структуры $\left(\psi_{0}, F_{0}\right)$, поэтому в отличие от двух особых точек для $\psi_{0}$ на $L$ мы имеем три особые точки на той же базе для псевдоторической структуры $(\psi, F)$ на поверхности $X$. Мы можем воспользоваться структурой двулистного накрытия $\pi$ для сравнения лагранжевых подмногообразий и лагранжевых слоений в $X$. В самом деле, построенная псевдоторическая структура на $X$ позволяет построить лагранжевы слоения на $X$ - достаточно выбрать произвольную гладкую функцию на базе псевдоторической структуры, как это делалось в работах [2], [6]. Одновременно выбор этой функции индуцирует лагранжево слоение на $\mathbb{C P}_{x}^{2}$, при этом двулистное накрытие $\pi$ индуцирует подъем лагранжевых подмногообразий из $\mathbb{C P}_{x}^{2}$. Если соответствующая линия уровня на базе $L$ не проходит через точку $[0: 1:-1]$, то 
прообразом лагранжева слоя будет пара лагранжевых торов, в противном случае слой поднимается до особого лагранжева слоя.

Закончим этот пример следующим естественным вопросом, ответ на который остается предметом будущей работы: возникают ли при этом новые типы неизотопных лагранжевых торов в двумерной квадрике в дополнение к стандартному типу Клиффорда и нестандартному типу Чеканова.

Благодарности. В заключение хочется выразить благодарность за ценные обсуждения С. Белёву, А. Миронову и А. Трепалину. Автор пользовался частичной финансовой поддержкой лаборатории алгебраической геометрии НИУ ВШЭ, а также Правительства РФ (грант аг. 11.G34.31.0023). Окончательная версия статьи выполнена при финансовой поддержке РНФ (соглашение № 14-21-00053 на выполнение научного проекта структурным подразделением организации - лабораторией алгебраической геометрии и ее приложений НИУ ВШЭ).

\section{Список литературы}

[1] M. Audin, Torus Actions on Symplectic Manifolds, Progress in Mathematics, 93, Birkhäuser, Basel, 2004.

[2] Н. А. Тюрин, ТМФ, 162:3 (2010), 307-333.

[3] Н. А. Тюрин, ТМФ, 167:2 (2011), 193-205.

[4] Н. А. Тюрин, УМН, 66:1(397) (2011), 185-186.

[5] P. Griffiths, J. Harris, Principles of Algebraic Geometry, John Wiley \& Sons, New York, 1994.

[6] С. А. Белев, Н. А. Тюрин, Матем. заметки, 87:1 (2010), 48-59.

Поступила в редакцию 28.03.2014, после доработки 5.09.2014 